\title{
COMPLETELY BOUNDED BANACH-MAZUR DISTANCE ${ }^{\dagger}$
}

\author{
by CHUN ZHANG
}

(Received 24th April 1995)

Analogous to the Banach-Mazur distance between Banach spaces, we study the completely bounded Banach-Mazur distance between operator spaces.

$$
d_{c b}(X, Y)=\inf \left\{\|T\|_{c b}\left\|T^{-1}\right\|_{c b}, \quad T: X \mapsto Y \text { is any linear isomorphism }\right\} .
$$

In many cases of Banach spaces and Hilbert spaces we show that the infimum is attained when $T$ is the identity map, and $X, Y$ have the same base space. This provides a machinery to compute and estimate $d_{c b}(X, Y)$. Later, using symmetric norming functions we construct counterexamples to show that distinct infinite dimensional homogeneous operator spaces may have finite cb-distance, and that two homogeneous Hilbertian operator spaces may not coincide even if they coincide over all 2-dimensional subspaces.

1991 Mathematics subject classification: primary 47D25

\section{Introduction}

In the study of the local theory of Branch spaces, people introduced various ways to measure the difference of structures between Banach spaces and their subspaces. Examples are the bounded approximation constant and the projection constant in [18]. Another Banach space constant which plays a considerably important role is the Banach-Mazur distance which is defined as follows.

Definition 1.1. Let $X, Y$ be Banach spaces, then the Banach-Mazur distance between them is defined to be

$$
d(X, Y)=\inf \left\{\|T\|\left\|T^{-1}\right\|: \quad X \mapsto Y \text { is a linear isomorphism }\right\}
$$

This distance makes some impact on operator space theory too. Paulsen's paper [13] contains an application of this distance to computations in operator spaces. There is an analogy of this distance in operator space theory. The completely bounded norm of a linear map between operator spaces $T: X \mapsto Y$ is defined to be

'This is part of author's Ph.D. thesis directed by Professor Vern Paulsen. The author wants to express his sincerest gratitude. 


$$
\|T\|_{c b}=\sup \left\{\left\|T_{n}\right\|: \quad n=1,2,3, \ldots\right\}
$$

where $T_{n}: M_{n}(X) \mapsto M_{n}(Y)$ is defined by $T_{n}\left(\left(x_{i j}\right)\right)=\left(T\left(x_{i j}\right)\right)$. The following defines the completely bounded Banach-Mazur distance which is briefly called cb-distance in the sequel.

Definition 1.2. For any operator spaces $E, F$, we define

$$
d_{c b}(E, F)=\inf \left\{\|T\|_{c b}\left\|T^{-1}\right\|_{c b}: \quad T: E \mapsto F \text { is any linear isomorphism }\right\}
$$

In [15], Pisier gave a general estimate that for any $n$ dimensional operator spaces $E, F, d_{c b}(E, F) \leq n$. This $n$ is the best constant that can possibly be obtained, since we shall see an example where the cb-distance between two $n$ dimensional operator spaces is exactly equal to $n$. Nevertheless, it is still tempting to compute the cb-distance more precisely for some particular classes of operator spaces. In Section 2, we show that for any two homogeneous operator spaces with the same underlying Banach spaces, the cb-distance of equation (1) is attained by the identity map when the two cb-norms are comparable. In Section 3 we study the case of homogeneous Hilbertian operator spaces. We are able to show that for any two such spaces the distance is attained by the identity operator. The theorems are applied to the computations of the cb-distance for several examples. Section 4 is a study of homogeneous Hilbertian operator spaces that are induced from symmetric norming functions. We define a distance $d\left(\Phi_{1}, \Phi_{2}\right)$ between symmetric norming functions, then using the results of Section 3 we prove that the cb-distance is equivalent to $d\left(\Phi_{1}, \Phi_{2}\right)$. This theorem provides a machinery for us to construct examples that clarify some puzzles about homogeneous operator spaces.

It's simple but worth remarking that for any operator spaces $X, Y, Z$,

$$
d_{c b}(X, Z) \leq d_{c b}(X, Y) d_{c b}(Y, Z)
$$

This immediately follows from the inequality for cb-norms

$$
\|A B\|_{c b} \leq\|A\|_{c b}\|B\|_{c b}
$$

In the category of all operator spaces, there is a special type of them called homogeneous operator spaces which was introduced by Pisier [14]. In this paper we study the cb-distance between homogeneous operator spaces. The notation $i_{X}$ is used very often to denote the identity map on $X$.

Definition 1.3. An operator space $X$ is said to be homogeneous if every $T: X \mapsto X$ satisfies $\|T\|_{e b}=\|T\|$. 


\section{The case of Banach spaces}

Proposition 2.1. Let $X$ be a Banach space which is assigned two homogeneous operator space structures, $X_{1}=\left(X,\left\{\|\|_{n}\right\}_{n=1}^{\infty}\right), X_{2}=\left(X,\left\{\|\|_{n}^{\prime}\right\}_{n=1}^{\infty}\right)$. Suppose that

$$
\left\|\left(x_{i j}\right)\right\|_{n}^{\prime} \leq\left\|\left(x_{i j}\right)\right\|_{n} \text { for all }\left(x_{i j}\right) \in M_{n}(X), \quad n=1,2,3 \ldots
$$

Then, $d_{c b}\left(X_{1}, X_{2}\right)=\left\|i_{X}\right\|_{c b}\left\|i_{X}^{-1}\right\|_{c b}$, where $i_{X}: X_{1} \mapsto X_{2}$ is the identity operator on $X$.

Proof. Let us first notice a fact that for any invertible linear map $T$ in $B\left(X_{1}, X_{2}\right)$, where $B\left(X_{1}, X_{2}\right)$ is the set of all bounded linear maps from $X_{1}$ into $X_{2}$, then $T$ is completely bounded and $\|T\|_{c b}=\|T\|$. To see this, consider the diagram

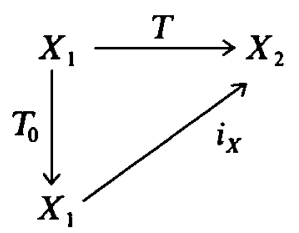

where $T_{0}$ is the same map as $T$ but regarded as a map from $X_{1}$ into itself. Whence we have $\|T\|_{c b}=\left\|i_{X} \circ T_{0}\right\|_{c b} \leq\left\|i_{X}\right\|_{c b}\left\|T_{0}\right\|_{c b}=\left\|T_{0}\right\|=\|T\|$, noticing that $i_{X}$ is completely contractive.

Now look at

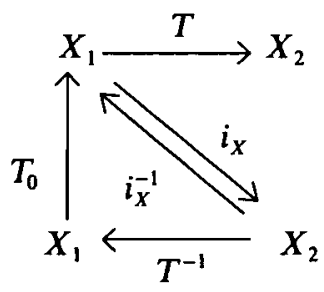

where $T_{0}$ bears the same sense as in the last diagram. Then, $\left\|i_{X}\right\|_{c b}\left\|i_{X}^{-1}\right\|_{c b} \leq\left\|i_{X}^{-1}\right\|_{c b}=$ $\left\|T_{0} \circ T^{-1}\right\| \leq\left\|T_{0}\right\|_{c b}\left\|T^{-1}\right\|_{c b}=\|T\|_{c b}\left\|T^{-1}\right\|_{c b}$.

Paulsen [13] introduced a numercial invariant $\alpha(X)$ for any Banach space $X$ which measures the difference between $\operatorname{MIN}(X)$ and $\operatorname{MAX}(X)$. The reader will also find the definitions of spaces $M I N(X)$ and $M A X(X)$ in [13].

$$
\alpha(X)=\sup \left\{\left\|\left(x_{i j}\right)\right\|_{\max }:\left\|\left(x_{i j}\right)\right\|_{\min } \leq 1\right\}
$$

We immediately see from the definition that 


$$
\alpha(X)=\left\|i_{X}\right\|_{c b}
$$

where $i_{X}: M I N(X) \mapsto M A X(X)$

Corollary 2.2. $d_{c b}(M I N(X), M A X(X))=\alpha(X)$ for any finite dimensional normed space $X$.

Proof. The operator spaces $M I N(X), M A X(X)$ are homogeneous since every map $T: E \mapsto M I N(X)$ must satisfy $\|T\|_{c b}=\|T\|$, and every map from a $M A X(X)$ also satisfies the same equation. Since $M I N(X)$ and $M A X(X)$ are homogeneous and comparable,

$$
d_{c b}(M I N(X), M A X(X))=\left\|i_{X}\right\|_{c b}\left\|i_{X}^{-1}\right\|_{c b}=\left\|i_{X}\right\|_{c b}=\alpha(X) .
$$

Proposition 2.1 enables us to calculate the cb-distance when two operator space norms are comparable. We don't know whether or not the conclusion of this proposition is true for any two homogeneous operator spaces norms on $X$. But if $X$ is a Hilbert space, we are able to show that it is true. This is done in the next section.

\section{The case of Hilbert spaces}

In this section, we do the computations of cb-distances between Hilbertian operator spaces (which means the underlying spaces are Hilbert spaces). Without loss of generality, we always suppose the two operator spaces, whose cb-distance we are computing, are on the same Hilbert space. The following theorem is both a tool of computation and a preparation for our main theorem.

Theorem 3.1. Let $H$ be an $n$ dimensional Hilbert space, $H_{1}, H_{2}$ be homogeneous operator spaces which have $H$ as the underlying space. Then

$$
d_{c b}\left(H_{1}, H_{2}\right)=\left\|i_{H}\right\|_{c b}\left\|i_{H}^{-1}\right\|_{c b},
$$

where $i_{H}$ is the identity operator on $\mathrm{H}$ but considered from $H_{1}$ to $H_{2}$.

Proof. Observe that if we decompose any fixed invertible operator $T: H_{1} \mapsto H_{2}$ as $T=T_{0} U$

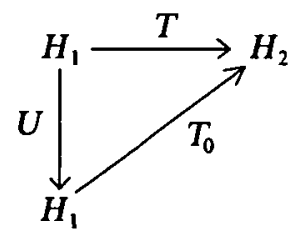


where $U$ is a unitary, then $\|T\|_{c b}=\left\|T_{0}\right\|_{c b}$. Indeed, $\|T\|=\left\|T_{0} U\right\|_{c b} \leq\left\|T_{0}\right\|_{c b}\|U\|_{c b}=$ $\left\|T_{0}\right\|_{c b}$, and conversely $\left\|T_{0}\right\|_{c b}=\left\|T U^{-1}\right\| \leq\|T\|_{c b}$. Similarly, $\left\|V T_{c b}=\right\| T \|_{c b}$ when $V$ is a unitary.

Now let $T=U|T|$ be a polar decomposition. Since $|T|$ is positive, it is diagonalizable, say, $|T|=V^{*} D V$, where $V$ is a unitary and $D=\operatorname{diag}\left\{d_{1}, d_{2}, \ldots, d_{n}\right\}$. Then $T=U V^{*} D V$, and $\|T\|_{c b}=\|D\|_{c b}$. Let $S$ be the permutation of the canonical basis of $H$ such that $S e_{1}=e_{2}, S e_{2}=e_{3}, \ldots, S e_{n-1}=e_{n}, S e_{n}=e_{1}$, then by permuting $D$ repeatedly, we get

$$
\begin{gathered}
\left(\frac{1}{n} \sum_{i=0}^{n-1} S^{i}\right) D=\frac{d_{1}+d_{2}+\cdots+d_{n}}{n} i_{H}, \\
\left(\frac{1}{n} \sum_{i=0}^{n-1} S^{i}\right) D^{-1}=\frac{d_{1}^{-1}+d_{2}^{-1}+\cdots+d_{n}^{-1}}{n} i_{H}^{-1} .
\end{gathered}
$$

By the geometric-arithmetic mean inequality

$$
\frac{\left(d_{1}+\cdots+d_{n}\right)\left(d_{1}^{-1}+\cdots+d_{n}^{-1}\right)}{n^{2}}=\frac{\sum_{i, j=1}^{n} d_{i} d_{j}^{-1}}{n^{2}} \geq \sqrt[n^{2}]{\prod_{i, j=1}^{n} d_{i} d_{j}^{-1}}=1
$$

Finally,

$$
\begin{aligned}
\left\|i_{H}\right\|_{c b}\left\|i_{H}^{-1}\right\|_{c b} & \leq \frac{\left(d_{1}+\cdots+d_{n}\right)\left(d_{1}^{-1}+\cdots+d_{n}^{-1}\right)}{n^{2}}\left\|i_{H}\right\|_{c b}\left\|i_{H}^{-1}\right\|_{c b} \\
& =\left\|\left(\frac{1}{n} \sum_{i=0}^{n-1} S^{i}\right) D\right\|_{c b}\left\|\left(\frac{1}{n} \sum_{i=0}^{n-1} S^{i}\right) D^{-1}\right\|_{c b} \\
& \leq\left\|\frac{1}{n} \sum_{i=0}^{n-1} S^{i}\right\|_{c b}\|D\|_{c b}\left\|\frac{1}{n} \sum_{i=0}^{n-1} S^{i}\right\|_{c b}\left\|D^{-1}\right\|_{c b} \\
& \leq\|D\|_{c b}\left\|D^{-1}\right\|_{c b} .
\end{aligned}
$$

It is known (see $[6,7])$ that for any $T \in \mathcal{B}(H)$, if $\|T\|_{H S}<\infty\left(\|\| \|_{H S}\right.$ denotes the Hilbert Schmidt norm), then $T$ is completely bounded when it is viewed as an element in any of the sets $C B\left(H_{\text {row }}, H_{\text {col }}\right), C B\left(H_{\text {col }}, H_{\text {row }}\right)$, (see [6]), $C B\left(M I N(H), H_{\text {row }}\right)$, $C B\left(H_{\text {row }}, M A X(H)\right.$ ), (see [9]), where $H_{\text {row }}$ and $H_{\text {col }}$ are the row and column Hilbert spaces, respectively. They are denoted by $R_{n}$ and $C_{n}$ respectively when $\operatorname{dim}(H)=n$. Furthermore, $\|T\|_{c b}=\|T\|_{H S}$ in all of these cases. In particular, $\left\|i_{H}\right\|_{c b}=\sqrt{n}$ if $H$ is $n$ dimensional.

\section{Corollary 3.2.}

$$
d_{c b}\left(R_{n}, C_{n}\right)=n
$$


Proof. Since $R_{n}, C_{n}$ are homogeneous (see [6]), $d_{c b}\left(R_{n}, C_{n}\right)=\left\|i_{H}\right\|_{c b}\left\|i_{H}^{-1}\right\|_{c b}=$ $\left\|i_{H}\right\|_{H S}\left\|i_{H}^{-1}\right\|_{H S}=\sqrt{n} \sqrt{n}=n$.

This result was also obtained independently by Mathes in [10]. The result of this corollary gives an example that the constant $n$ in Pisier's theorem is attainable. Other similar computations will yield $d_{c b}\left(M I N(H), R_{n}\right)=d_{c b}\left(M I N(H), C_{n}\right)=\sqrt{n}$.

A more general class of homogeneous Hilbertian operator spaces can be obtained by the idea of complex interpolation of Banach spaces. The reader is referred to $[1,14]$ for the definitions and basic properties of interpolations of Banach spaces and operator spaces respectively. For a compatible pair of operator spaces $E_{0}$ and $E_{1}$, following Pisier [14] we use $\left(E_{0}, E_{1}\right)_{0}$ as the notation for the interpolated spaces between $E_{0}$ and $E_{1}$.

It is known that if $E_{0}$ and $E_{1}$ are homogeneous with the same underlying Banach space $E$, then $\left(E_{0}, E_{1}\right)_{0}$ is homogeneous for all $\theta$. Indeed, let $T:\left(E_{0}, E_{1}\right) \mapsto\left(E_{0}, E_{1}\right)$ be any completely bounded map. Let $\left\|T_{0}\right\|_{c b}$ denote the cb-norm of $T$ when it is viewed as $T:\left(E_{0}, E_{1}\right)_{0} \mapsto\left(E_{0}, E_{1}\right)_{0}$. Then we have

$$
\left\|T_{0}\right\|_{c b} \leq\left(\left\|T_{0}\right\|_{c b}\right)^{0}\left(\left\|T_{1}\right\|_{c b}\right)^{1-0}=\|T\|^{0}\|T\|^{1-0}=\|T\| .
$$

Where $T_{0}, T_{1}$ are both $T$ regarded as $T_{0}: E_{0} \mapsto E_{0}, T_{1}: E_{1} \mapsto E_{1}$.

Notation. Let $T: X \mapsto Y$ be a completely bounded map, in the following discussion, we use $\|T\|_{c b(X, Y)}$ for its cb-norm when it is necessary to make it clear where the cb-norm is taken.

Proposition 3.3. Let $H_{n}$ be an $n$ dimensional Hilbert space. For every $\theta \in[0,1]$,

(i) $d_{c b}\left(R_{n},\left(R_{n}, C_{n}\right)_{0}\right)=d_{c b}\left(C_{n}\left(R_{n}, C_{n}\right)_{1-0}\right)=n^{0}$.

(ii) $d_{c b}\left(M I N\left(H_{n}\right),\left(M I N\left(H_{n}\right), M A X\left(H_{n}\right)\right)_{0}\right)=\alpha\left(H_{n}\right)^{0}$; $d_{c b}\left(M A X\left(H_{n}\right),\left(M I N\left(H_{n}\right), M A X\left(H_{n}\right)\right)_{1-0}\right)=\alpha\left(H_{n}\right)^{0}$.

Proof. $\quad d_{c b}\left(R_{n},\left(R_{n}, C_{n}\right)_{0}\right)=\left\|i_{H_{n}}\right\|_{c b}\left\|i_{H_{n}}^{-1}\right\|_{c b}$ $\leq\left\|i_{H_{n}}\right\|_{c b\left(R_{n}, R_{n}\right)}^{1-0}\left\|i_{H_{n}}\right\|_{c b\left(R_{n}, C_{n}\right)}^{0}\left\|i_{H_{n}}^{-1}\right\|_{c b\left(C_{n}, C_{n}\right)}^{1-0}\left\|i_{H_{n}}^{-1}\right\|_{c b\left(C_{n}, R_{n}\right)}^{0}$ $=1 \cdot n^{\frac{1}{2}} \cdot 1 \cdot n^{\frac{0}{2}}=n^{0}$.

Similarly,

$$
d_{c b}\left(\left(R_{n}, C_{n}\right)_{0}, C_{n}\right) \leq n^{1-0}
$$

Thus $n=d_{c b}\left(R_{n}, C_{n}\right) \leq d_{c b}\left(R_{n}\left(R_{n}, C_{n}\right)_{0}\right) d_{c b}\left(\left(R_{n}, C_{n}\right)_{0}, C_{n}\right) \leq n^{0} n^{1-0}=n$. Thus we conclude that $d_{c b}\left(R_{n}\left(R_{n}, C_{n}\right)_{0}\right)=n^{0}$. By replacing $R_{n}$ by $M I N\left(H_{n}\right)$, and $C_{n}$ by $M A X\left(H_{n}\right)$ in the proof of (i) we get a proof of (ii).

Proposition 3.4. For all $\theta \in[0,1]$,

$$
d_{c b}\left(C_{n},\left(M I N\left(H_{n}\right), M A X\left(H_{n}\right)\right)_{0}\right)=d_{c b}\left(R_{n},\left(M I N\left(H_{n}\right), M A X\left(H_{n}\right)\right)_{0}\right)=\sqrt{n} .
$$


Proof.

$$
\begin{gathered}
d_{c b}\left(R_{n}\left(\operatorname{MIN}\left(H_{n}\right), M A X\left(H_{n}\right)\right)_{0}\right)=\left\|i_{H_{n}}\right\|_{c b}\left\|i_{H_{n}}^{-1}\right\|_{c b} \\
\leq\left\|i_{H_{n}}\right\|_{c b\left(R_{n}, M I N\left(H_{n}\right)\right)}^{1-\theta}\left\|i_{H_{n}}\right\|_{c b\left(R_{n} M A X\left(H_{n}\right)\right)}^{0}\left\|i_{H_{n}}^{-1}\right\|_{c b\left(M I N\left(H_{n}\right), R_{n}\right)}^{1-0}\left\|i_{H_{n}}^{-1}\right\|_{c b\left(M A X\left(H_{n}\right), R_{n}\right)}^{0} \\
=1 \cdot n^{\frac{0}{2}} \cdot n^{\frac{1-0}{2}} \cdot 1=\sqrt{n} .
\end{gathered}
$$

Similarly, $d_{c b}\left(C_{n},\left(M I N\left(H_{n}\right), M A X\left(H_{n}\right)\right)_{0}\right) \leq \sqrt{n}$.

If $d_{c b}\left(R_{n},\left(M I N\left(H_{n}\right), M A X\left(H_{n}\right)\right)_{0}\right)<\sqrt{n}$, then

$$
\begin{gathered}
n=d_{c b}\left(R_{n}, C_{n}\right) \leq d_{c b}\left(R_{n}\left(M I N\left(H_{n}\right), M A X\left(H_{n}\right)_{0}\right)\right) d_{c b}\left(\left(M I N\left(H_{n}\right), M A X\left(H_{n}\right)_{0}, C_{n}\right)\right) \\
<\sqrt{n} \sqrt{n}=n,
\end{gathered}
$$

a contradiction which implies that $d_{c b}\left(R_{n},\left(M I N\left(H_{n}\right), M A X\left(H_{n}\right)\right)_{0}\right)=\sqrt{n}$.

In exactly the same way we can prove the other equality.

We turn our attention now to the cb-distance between infinite dimensional Hilbertian operator spaces. In the previous two propositions, if the dimension $n$ is replaced by $\infty$, then all the cb-distances turn to $\infty$. This gives rise to a natural question whether or not the cb-distance between any distinct infinite dimensional homogeneous Hilbertian operator spaces is infinity. We will give a counterexample in the next section.

If $H$ is an infinite dimensional homogeneous Hilbertian operator space, $H_{1}$ is a subspace of the same dimension, then $H, H_{1}$ are completely isometric. Therefore if $H$ and $K$ are homogeneous with the same underlying space, and if $i$ is the identity operator $i: H \mapsto K$, then $\left\|\left.i\right|_{H_{1}}\right\|_{c b}=\|i\|_{c b}$.

Lemma 3.5. Let $H, K$ be homogeneous operator spaces with the same underlying Hilbert space, $K_{1} \subset K$ be a subspace of the same dimension as $K$. Let $P$ be the orthogonal projection of $H$ onto $K_{1}$, then $\|i\|_{c b(H, K)}=\|P\|_{c b(H, K)}$.

Proof. Obviously $\|P\|_{c b} \leq\|i\|_{c b}$. Now let $H_{1}=i^{-1}\left(K_{1}\right)$, then

$$
\|i\|_{c b}=\left\|\left.i\right|_{H_{1}}\right\|_{c b}=\left\|\left.P\right|_{H_{1}}\right\|_{c b} \leq\|P\|_{c b} .
$$

Thus $\|P\|_{c b}=\|i\|_{c b}$.

Theorem 3.6. Let $H_{1}, H_{2}$ be any homogeneous operator spaces with the same underlying infinite dimensional Hilbert space $H$, then

$$
d_{c b}\left(H_{1}, H_{2}\right)=\left\|i_{H}\right\|_{c b}\left\|i_{H}^{-1}\right\|_{c b} .
$$


Proof. For any invertible operator $T \in \mathcal{B}(H)$, there is a polar decomposition $T=U \cdot|T|$, where $U: H_{2} \mapsto H_{2}$ is a unitary. Automatically $\||T|\|_{c b}=\|T\|_{c b}$. So we reduce to the case when $T$ is positive.

Let $\Gamma: C^{*}(T) \mapsto C(\sigma(T))$ be the Gelfand transform, where $C^{*}(T)$ denotes the $C^{*}$-algebra generated by $T, \sigma(T)$ is the spectrum of $T$.

For any fixed $\epsilon>0$, let $\sigma(T)=\bigcup_{i=1}^{n} A_{i}$ be a partition of $\sigma(T)$, such that the diameter of each $A_{i}$ is smaller than $\epsilon$. Letting $\chi_{A_{i}}$ be the characteristic function of $A_{i}$ we define

$$
\phi(z)=\sum_{i=1}^{n} z_{i} \chi_{A_{i}}
$$

where $z_{i}$ is a point in $A_{i}$, and

$$
S=\Gamma^{-1}(\phi),
$$

then $\|T-S\|=\|z-\phi\| \leq \epsilon$. Notice that $S$ is a diagonalizable operator with eigenvalues $z_{1}, z_{2}, \ldots, z_{n}$. If $K_{i}$ is the corresponding eigenspace of $z_{i}, i=1,2, \ldots, n$, then $S=\sum_{i=1}^{n} z_{i} P_{K_{i}}$ and $S^{-1}=\sum_{i=1}^{n} z_{i}^{-1} \tilde{P}_{K_{i}}$. Here $\tilde{P}_{K_{i}}$ is the same operator as $P_{K_{1}}$, but the former is considered to be $H_{2} \mapsto H_{1}$. By the continuity of inversion we can suppose $\left\|T^{-1}-S^{-1}\right\| \leq \epsilon$. Among $K_{1}, K_{2}, \ldots K_{n}$ there is at least one $K_{i}$ such that $\operatorname{dim}\left(K_{i}\right)=\operatorname{dim}(H)$, say $K_{i}$, then

$$
\|S\|_{c b}\left\|S^{-1}\right\|_{c b} \geq\left\|z_{i} P_{K_{i}}\right\|_{c b}\left\|z_{i}^{-1} \tilde{P}_{K_{i}}\right\|_{c b}=\left\|P_{K_{i}}\right\|_{c b}\left\|\tilde{P}_{K_{i}}\right\|_{c b}=\left\|i_{H}\right\|_{c b}\left\|i_{H}^{-1}\right\|_{c b}
$$

therefore

$$
\|T\|_{c b}\left\|T^{-1}\right\|_{c b} \geq\left(\|S\|_{c b}-\epsilon\right)\left(\left\|S^{-1}\right\|_{c b}-\epsilon\right) \geq(1-\epsilon)^{2}\left\|i_{H}\right\|_{c b}\left\|i_{H}^{-1}\right\|_{c b}
$$

Corollary 3.7. Two homogeneous Hilbertian operator spaces $H_{1}, H_{2}$ are completely isometric if and only if $d_{c b}\left(H_{1}, H_{2}\right)=1$.

\section{Homogeneous operator spaces induced by symmetric norming functions}

In this section we take a look at the homogeneous Hilbertian operator spaces induced from symmetric norming functions. These operator spaces were first introduced by $B$. Mathes and V. Paulsen (see [11]). We use the idea to construct the counterexamples referred to in the last section. We also prove a theorem which estimates the cb-distance between any two of these spaces.

Apart from the usual operator norm, there are various other norms that can be defined on $\mathcal{B}(H)$. We are interested in a particular class of these called symmetric norming functions. We restrict these functions to be defined on the finite rank operators only. 
Definition 4.1. A norm $\Phi$ on $\mathcal{F}(H)$ is called a symmetric norming function if

$$
\Phi(A B C) \leq\|A\| \cdot \Phi(B) \cdot\|C\| \quad \text { for all } A, C \in \mathcal{B}(H), B \in \mathcal{F}(H)
$$

where \|\| is the usual operator norm, and $\mathcal{F}(H)$ is the set of all finite rank operators on $H$.

Note. First, the definition immediately implies that $\Phi(U T V)=\Phi(T)$ when $U, V$ are unitaries, and vice versa. i.e. if $\Phi(U T V)=\Phi(T)$ for all unitaries $U$, $V$ and finite rank operator $T$, then $\Phi$ is a symmetric norming function. (See [7, Theorem $3.1 \&$ Corollary 3.1] for a justification). Secondly, the natural domain of $\Phi,\{T \in \mathcal{B}(H): \Phi(T)<\infty\}$ is an ideal of $\mathcal{B}(H)$.

If $H_{1}, H_{2}$ are homogeneous Hilbertian operator spaces with the same underlying space $H$, then the cb-norm with respect to $C B\left(H_{1}, H_{2}\right)$ is a symmetric norming function on $\mathcal{B}(H)$. Indeed, for any $A, C \in \mathcal{B}(H), B \in C B\left(H_{1}, H_{2}\right)$,

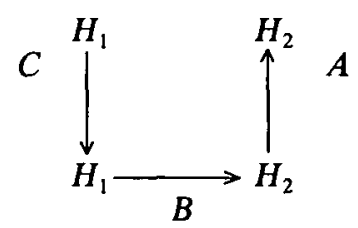

$\|A B C\|_{c b} \leq\|A\|_{c b}\|B\|_{c b}\|C\|_{c b}=\|A\|\|T\|_{c b}\|C\|$.

If $H$ is an $l^{2}$ space, $T$ is a compact operator on $H$ then a suitable choice of unitaries $U, V$ can result in $U T V$ being a diagonal operator or even a positive diagonal operator with the diagonal elements in non-increasing order. And notice that if $T \in C B\left(H_{1}, H_{2}\right), U T V=\operatorname{diag}\left\{\alpha_{1}, \alpha_{2}, \ldots\right\}$ in non-increasing order, then $\|T\|_{c b}=\left\|\operatorname{diag}\left\{\alpha_{1}, \alpha_{2}, \ldots\right\}\right\|_{c b}$. So the symmetric norming function \|\|$_{c b}$ is completely determined by a "unitarily invariant" norm on an infinite sequence space.

Conversely, from any symmetric norming function $\Phi$ on $\mathcal{B}(H)$, we can induce a homogeneous Hilbertian operator space norm on $H$ in the following way (see [11]), for any $\left(x_{i j}\right) \in M_{n}(H)$

$$
\left(\left\|\left(x_{i j}\right)\right\|_{\Phi}=\sup \left\{\left\|\left(T x_{i j}\right)\right\|_{\max }: \Phi(T) \leq 1\right\}\right.
$$

We denote $H$ with this matrix norm structure $H_{\Phi, \max }$. It is known that $H_{\Phi, \max }$ is homogeneous (see [11]).

By the above remark the cb-norm in the sense of $C B\left(H_{\Phi, \max }, H_{\max }\right)$ is a symmetric norming function on $H$, we denote it by $\Phi_{c b}$, i.e.

$$
\Phi_{c b}(T)=\|T\|_{c b} \quad \text { for } T \in C B\left(H_{\Phi_{, \max }}, H_{\max }\right) \text {. }
$$

Theorem 3.2 of [11] states that there is a constant $C>0$ such that 


$$
C \Phi(T) \leq \Phi_{c b}(T) \leq \Phi(T) \text { for all finite rank } T
$$

It is natural to study the behaviour of $d_{c b}\left(H_{\Phi_{1}, \max }, H_{\Phi_{2}, \max }\right)$. As one may expect it is related to the distance between $\Phi_{1}$ and $\Phi_{2}$.

Definition 4.2. Let $\Phi_{1}, \Phi_{2}$ be symmetric norming functions, we define

$$
d\left(\Phi_{1}, \Phi_{2}\right)=\sup _{T \in \mathcal{F}(H)} \frac{\Phi_{1}(T)}{\Phi_{2}(T)} \cdot \sup _{T \in \mathcal{F}(H)} \frac{\Phi_{2}(T)}{\Phi_{1}(T)}
$$

Theorem 4.3. Let $\Phi_{1}, \Phi_{2}$ be symmetric norming functions, then there is a constant $C>0$ such that

$$
C d\left(\Phi_{1}, \Phi_{2}\right) \leq d_{c b}\left(H_{\Phi_{1}, \max }, H_{\Phi_{2}, \max }\right) \leq d\left(\Phi_{1}, \Phi_{2}\right)
$$

Proof. As we know, $d_{c b}\left(H_{\Phi_{1}, \max }, H_{\Phi_{2}, \max }\right)=\left\|i_{H}\right\|_{c b\left(\Phi_{1}, \Phi_{2}\right)}\left\|i_{H}^{-1}\right\|_{c b\left(\Phi_{2}, \Phi_{1}\right)}$. Suppose

$$
\sup _{T \in \mathcal{F}(h)} \frac{\Phi_{1}(T)}{\Phi_{2}(T)}=k
$$

then $\left\{T \in \mathcal{F}(H): \Phi_{2}(T) \leq 1\right\} \subseteq\left\{T \in \mathcal{F}(H): \Phi_{1}(T) \leq k\right\}$. Thus

$$
\begin{aligned}
\sup \left\{\left\|\left(T x_{i j}\right)\right\|_{\max }: \quad \Phi_{2}(T) \leq 1\right\} & \leq \sup \left\{\left\|\left(T x_{i j}\right)\right\|_{\max }:\right. & \left.\Phi_{1}(T) \leq k\right\} \\
& =k \sup \left\{\left\|\left(T x_{i j}\right)\right\|_{\max }:\right. & \left.\Phi_{1}(T) \leq 1\right\} .
\end{aligned}
$$

So $\left\|\left(x_{i j}\right)\right\|_{\Phi_{2}, \max } \leq k\left\|\left(x_{i j}\right)\right\|_{\Phi_{1}, \max }$ for any $\left(x_{i j}\right) \in M_{n}(H)$. This means

$$
\left\|i_{H}\right\|_{c b\left(\Phi_{1}, \Phi_{2}\right)} \leq k=\sup _{T \in \mathcal{F}(H)} \frac{\Phi_{1}(T)}{\Phi_{2}(T)} .
$$

Similarly,

$$
\left\|i_{H}^{-1}\right\|_{c b\left(\Phi_{2}, \Phi_{1}\right)} \leq \sup _{T \in \mathcal{F}(H)} \frac{\Phi_{2}(T)}{\Phi_{1}(T)}
$$

Thus $d_{c b}\left(H_{\Phi_{1}, \max }, H_{\Phi_{2}, \max }\right) \leq d_{c b}\left(\Phi_{1}, \Phi_{2}\right)$.

For the other inequality, take any $T \in \mathcal{F}(H)$ and look at the diagram

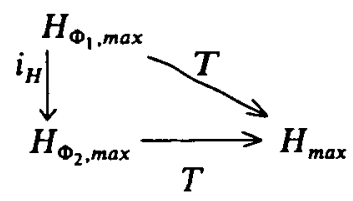


Then

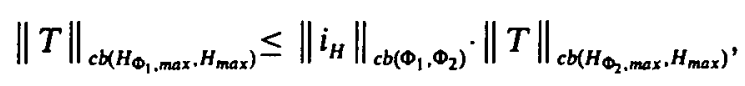

i.e.

$$
\left(\Phi_{1}\right)_{c b}(T) \leq\left\|i_{H}\right\|_{c b\left(\Phi_{1}, \Phi_{2}\right)} \cdot\left(\Phi_{2}\right)_{c b}(T)
$$

or

$$
\left\|i_{H}\right\|_{c b\left(\Phi_{1}, \Phi_{2}\right)} \geq \frac{\left(\Phi_{1}\right)_{c b}(T)}{\left(\Phi_{2}\right)_{c b}(T)} \geq \frac{C_{1} \Phi_{1}(T)}{\Phi_{2}(T)}
$$

for all $T \in \mathcal{F}(H)$ and for some $C_{1}>0$. Similarly

$$
\left\|i_{H}^{-1}\right\|_{c b\left(\Phi_{2}, \Phi_{1}\right)} \geq \frac{C_{2} \Phi_{2}(T)}{\Phi_{1}(T)}
$$

for all $T \in \mathcal{F}(H)$ and for some $C_{2}>0$. Let $C=C_{1} C_{2}$, then

$$
\left\|i_{H}\right\|_{c b\left(\Phi_{1}, \Phi_{2}\right)}\left\|i_{H}^{-1}\right\|_{c b\left(\Phi_{2}, \Phi_{1}\right)} \geq C \sup \frac{\Phi_{1}(T)}{\Phi_{2}(T)} \cdot \sup \frac{\Phi_{2}(T)}{\Phi_{1}(T)}
$$

where the sup's are over all $T \in \mathcal{F}(H)$. Consequently we have

$$
C d\left(\Phi_{1}, \Phi_{2}\right) \leq d_{c b}\left(H_{\Phi_{1}, \max }, H_{\Phi_{2}, \max }\right)
$$

This theorem generalizes [11, Theorem 3.2], and it has the following applications in constructing examples as well.

Example. This example gives infinite dimensional homogeneous Hilbertian operator spaces whose cb-distances to each other are finite. For every $r \in(0,1)$, we define a symmetric norming function via, for any $\left(\lambda_{1}, \lambda_{2}, \lambda_{3}, \ldots\right), \lambda_{1} \geq \lambda_{2} \geq \lambda_{3} \geq \cdots \geq 0$, let

$$
\phi_{r}\left(\lambda_{1}, \lambda_{2}, \lambda_{3}, \ldots\right)=\sup _{n}\left\{\frac{\lambda_{1}+\lambda_{2}+\cdots+\lambda_{n}}{1+r+\cdots+r^{n-1}}\right\} \text {. }
$$

Then all $\phi_{r}$ 's are equivalent to the $l^{1}$ norm, since

$$
\sum_{i=1}^{\infty} \lambda_{i} \geq \frac{\lambda_{1}+\lambda_{2}+\cdots+\lambda_{n}}{1+r+\cdots r^{n-1}} \geq \frac{\sum_{i=1}^{n} \lambda_{i}}{\frac{1}{1-r}}=(1-r) \sum_{i=1}^{n} \lambda_{i} .
$$

Hence 


$$
(1-r)\|T\|_{1} \leq \phi_{r}(T) \leq\|T\|_{1}, \quad \text { for all } T \text {. }
$$

Now if $r_{1}>r_{2}$, then

$$
d_{c b}\left(H_{\phi_{r_{1}}, \max }, H_{\phi_{r_{2}}, \max }\right)=\left\|i_{H}\right\|_{c b} .
$$

By Theorem 4.3 we see that

$$
\left\|i_{H}\right\|_{c b} \leq \frac{1}{1-r}<+\infty
$$

On the other hand, $\left\{H_{\phi_{r}, \max }, r \in(0,1)\right\}$ is not a singleton, i.e. $H_{\phi_{r}, \max }$ are not all identical. Otherwise,

$$
H_{\phi_{1}, \max }=\bigvee_{r \in(0,1)} H_{\phi_{r}, \max }=H_{\phi_{r}, \max }
$$

where the symbol $\bigvee$ means to sup the norms of all $H_{\phi_{r} \text { max }}$. We have reached a contradiction since $\phi_{1}\left(\lambda_{1}, \lambda_{2}, \ldots\right)=\lambda_{1}$, i.e. $\phi_{1}$ is the usual operator norm on $H$. But it is easily seen that $H_{\phi_{1}, \max }=M A X(H)$. Indeed, for every $\left(x_{i j}\right) \in M_{n}(H)$

$$
\begin{aligned}
& \left\|\left(x_{i j}\right)\right\|_{\phi_{1}, \max }=\sup \left\{\left\|\left(T x_{i j}\right)\right\|_{\max }:\|T\| \leq 1\right\} \\
& \leq \sup \left\{\left\|\left(T x_{i j}\right)\right\|_{\max }: \quad T \text { is a unitary }\right\} \\
& =\left\|\left(x_{i j}\right)\right\|_{\max } \text {. }
\end{aligned}
$$

For a Banach space $X$, it is well known that if all its two dimensional subspaces are isometric to a Hilbert space, then $X$ is a Hilbert space. Here is a similar (but not very much the same) question in the case of operator space: if $H_{1}, H_{2}$ are homogeneous on Hilbert space $H$, and if the identity map $i_{H}: H_{1} \mapsto H_{2}$ is a complete isometry when restricted to each two dimensional subspace, are $H_{1}$ and $H_{2}$ identical as operator spaces? The next example answers this question.

Proposition 4.4. Let $\Phi_{1}, \Phi_{2}$ be symmetric norming functions, and fix $n$. If $\Phi_{1}(T)=$ $\Phi_{2}(T)$ for all operators $T$ with rank $\leq n$. Then two induced operator space structures $H_{\Phi_{1}, \max }, H_{\Phi_{2}, \max }$ agree on all $n$ dimensional subspaces.

Proof. Suppose $K$ is any $n$ dimensional subspace of $H,\left(x_{i j}\right) \in M_{n}(K)$, then 


$$
\begin{aligned}
\left\|\left(x_{i j}\right)\right\|_{\Phi_{1}, \max } & =\sup \left\{\left\|\left(T x_{i j}\right)\right\|_{\max }:\right. & \left.\Phi_{1}(T) \leq 1\right\} & \\
& =\sup \left\{\left\|\left(T x_{i j}\right)\right\|_{\max }:\right. & \Phi_{1}(T) \leq 1, & \operatorname{rank}(T) \leq n\} \\
& =\sup \left\{\left\|\left(T x_{i j}\right)\right\|_{\max }:\right. & \Phi_{2}(T) \leq 1, & \operatorname{rank}(T) \leq n\} \\
& =\sup \left\{\left\|\left(T x_{i j}\right)\right\|_{\max }:\right. & \left.\Phi_{2}(T) \leq 1\right\} & \\
& =\left\|\left(x_{i j}\right)\right\|_{\Phi_{2}, \max } & &
\end{aligned}
$$

Note that the second equality is true because $K$ is $n$ dimensional, and we can consider the decomposition $H=K \oplus K^{\perp}$.

Example. Let $\Phi_{1}, \Phi_{2}$ be

$$
\begin{aligned}
& \Phi_{1}\left(\lambda_{1}, \lambda_{2}, \ldots\right)=\lambda_{1}+\lambda_{2} \\
& \Phi_{2}\left(\lambda_{1}, \lambda_{2}, \ldots\right)=\sum_{i=1}^{\infty} \lambda_{i}
\end{aligned}
$$

for all $\lambda_{1} \geq \lambda_{2} \geq \cdots \geq 0$. Then clearly $\Phi_{1}(T)=\Phi_{2}(T)$ if $T$ is rank 2 . Thus by the previous lemma, $H_{\Phi_{1}, \max }, H_{\Phi_{2}, \max }$ are identical on all two dimensional subspaces. But $\Phi_{1}$, $\Phi_{2}$ are not equivalent norms, hence by Theorem $4.3, H_{\Phi_{1}, \max }, H_{\Phi_{2}, \max }$ are not even completely bounded equivalent.

This example is easily generalized from dimension 2 to dimension $n$.

\section{REFERENCES}

1. J. Bergh and J. LofSTrom, Interpolation spaces (Springer-Verlag, Berlin, Heidelberg, New York, 1976).

2. D. P. Blecher, The standard dual of an operator space, Pacific J. Math. 153 (1992), $15-30$.

3. D. P. Blecher and V. I. PAulsen, Tensor products of operator spaces, J. Funct. Anal. 99 (1991), 262-292.

4. R. G. Douglas, Banach Algebra Techniques in Operator Theory (Academic Press, 1972).

5. E. G. EFrros, Advances in quantized functional analysis, in Proceedings, International Congress of Mathematicians Berkeley, 1986 (American Mathematical Society, 1987).

6. E. G. EfFros and Z. J. RuAN, Self-duality for the Haagerup tensor product and Hilbert space factorization, J. Funct. Anal. 100 (1991), 157-184.

7. I. C. GoBerg and M. G. KReIN, Introduction to the Theory of Linear Non-selfadjoint Operators (Transl. Math. Monographs 18 (1969)).

8. R. V. KADISON and J. R. RINGROSE, Fundamentals of the Theory of Operator Algebras (Academic Press 100-I (1983)).

9. B. Mathes, A completely bounded view of Hilbert-Schmidt operators, Houston Math. J. 17 (1991), 404-418. 
10. B. Mathes, Characterizations of row and column Hilbert spaces, J. London Math. Soc., to appear.

11. B. Mathes and V. I. Paulsen, Operator ideals and operator spaces, Proc. Amer. Math. Soc, to appear.

12. V. I. PAULSEN, Completely bounded maps and dilations (Pitman Research Notes in Math. Series 146).

13. V. I. PAULSEN, Representations of function algebras, abstract operator spaces, and Banach space geometry, J. Funct. Anal. 109 (1992), 113-129.

14. G. PISIER, The operator Hilbert space and complex interpolation, preprint.

15. G. PISIER, On the local theory of operator spaces, preprint.

16. G. PISIER, The operator Hilbert space $O H$, complex interpolation and tensor norms (Lecture Notes, Texas A \& M University).

17. Z.-J. Ruan, Subspaces of C*-algebras, J. Funct. Anal. 76 (1988), 217-230.

18. P. WoJTASzczYK, Banach Spaces for Analysts (Cambridge University Press, 1991).

DepartMENT OF MATHematics

THE UNIVERSITY OF HOUSTON

HousToN, TX 77204

U.S.A. 